\title{
Understanding EGFR Signaling in Breast Cancer and Breast Cancer Stem Cells: Overexpression and Therapeutic Implications
}

\author{
Ibrahim O Alanazi ${ }^{1 *}$, Zahid Khan²
}

\begin{abstract}
Epidermal growth factor receptors (EGFRs/HERs) and downstream signaling pathways have been implicated in the pathogenesis of several malignancies including breast cancer and its resistance to treatment with chemotherapeutic drugs. Consequently, several monoclonal antibodies as well as small molecule inhibitors targeting these pathways have emerged as therapeutic tools in the recent past. However, studies have shown that utilizing these molecules in combination with chemotherapy has yielded only limited success. This review describes the current understanding of EGFRs/HERs and associated signaling pathways in relation to development of breast cancer and responses to various cancer treatments in the hope of pointing to improved prevention, diagnosis and treatment. Also, we review the role of breast cancer stem cells (BCSCs) in disease and the potential to target these cells.
\end{abstract}

Keywords: Breast cancer - epithelial growth factor receptors - breast cancer stem cells - MAbs - TKI

Asian Pac J Cancer Prev, 17 (2), 445-453

\section{Introduction}

Breast cancer is one of the most common cancers among women (Dimitrakopoulos et al., 2015). The possibility of having breast cancer is high if there is a family history of breast cancer, especially if a close relative such as a mother, sister or daughter has been diagnosed with breast cancer at a premenopausal age (Ruddon, 2007; Shiovitz and Korde, 2015). There were an estimated 494,000 new cases of breast cancer diagnosed in Europe and about 1.67 million worldwide in 2012 (Lyon and France, 2013). The occurrence of breast cancer around the world is predicted to increase to 2.3 million by 2030 , although mortality rates in western countries are declining due to earlier detection and effective adjuvant treatment (Twelves and Stebbing, 2012).

The contribution of genetic factors in breast cancer is estimated to be about $5 \%$ of all cases but approximately $25 \%$ of cases are diagnosed with an early onset of the disease before the age of 30 years (Miki et al., 1994). Aberrations in several signaling pathways have been pathologically implicated in the development of carcinomas of the breast. Among them, the pathway involving dysregulation of the human epidermal growth factor receptor (HER) family of proteins plays crucial role in breast cancer. For instance, the over expression of epidermal growth factor receptor, EGFR (HER1) and HER2 genes, which are members of HER family receptors, have been causally implicated in breast cancer (Ruddon, 2007; Bouchalova et al., 2009). HER 1 expression is detected in about $40 \%$ of breast cancer (Bouchalova et al., 2009) while amplification or the over expression of the HER2 gene occurs in up to 25$30 \%$ of patients with breast cancer (Slamon et al., 1987; Vandevijver et al., 1988; Baselga, 2001; Basu, 2008). Over expression of HER1 and HER2, which are preferred interacting partners for dimerization in breast cancer, is associated with a poorer clinical prognosis and predicts a bad response to endocrine therapy (Valdehita et al., 2012).

\section{HER 1/HER2 Receptors and Activated Pathways}

HER1 is a single chain transmembrane polypeptide protein that consists of three domains. These domains are the extracellular, transmembrane and intracellular tyrosine kinase domains. The extracellular domain permits binding of ligands to activate the receptor for intracellular signaling. The transmembrane domain engages in homodimerization between HER1 receptors and heterodimerization with HER2, which is a preferred partner for dimerization in breast cancer as depicted in figure 1. The intracellular tyrosine kinase domain has a carboxyl-terminal tail containing tyrosine autophosphorylation sites (Kamath and Buolamwini, 2006; Huang and $\mathrm{Fu}, 2015$ ). The phosphorylation of specific tyrosine residues within the activated receptor creates binding sites for adaptor proteins that contain Src homology 2 and phosphotyrosine binding domains (PTB) (Kamath and Buolamwini, 
2006; Normanno et al., 2006). These proteins activate a large number of signal transduction molecules such as protein kinase B (PKB or AKT), tyrosine kinase Src, c-Jun Kinase, stress-activated protein kinase and signal transducer and activator of transcription (STATs) (Kamath and Buolamwini, 2006) and subsequent stimulation of downstream signaling pathways such as PI3K/Akt, Ras/ Raf/Mek/Erk, PLC $\gamma$ 1/PKC, and STAT pathways (Brand et al., 2011; Huang and Fu, 2015) as can be seen in figure 2. Activation of these signaling cascades may lead to a variety of cellular responses such as proliferation, survival, differentiation and cell motility.

\section{HERI and HER2 in cancer}

HER 1 has been proposed as a potential anticancer target since 1980s. Enormous clinical data indicate the implication of HER 1 and HER2 in carcinogenesis and have supported the validity of targeting HER 1 and HER2 for cancer chemotherapy. The expression of HER 1 ranges from 40,000-100,000 receptors per cell in normal cells (Carpenter and Cohen, 1979), while the expression of HER 1 in common solid tumors including breast cancer is up to 2 million receptors per cell (Ennis

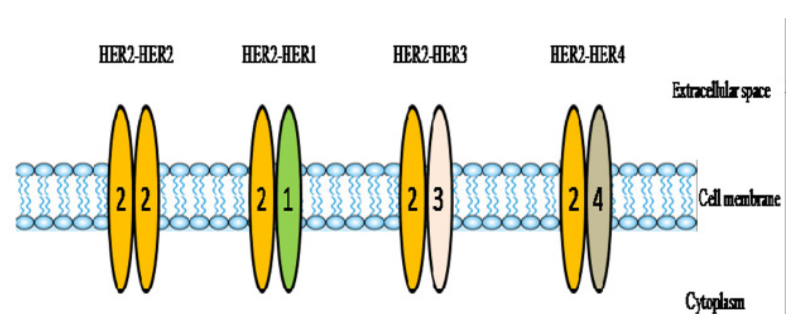

Figure 1. HER2 is the Preferred Dimerization Partner for the other HERs. Ligands binding to HERs except HER2 which has no known ligands induces the formation of receptor homo and heterodimers et al., 1991). The dysregulation of HER1 signaling pathway leads to carcinogenesis by various mechanisms including receptor and ligand overexpression, deficiency of specific phosphatases, decreased receptor turnover and altered dimerization process (Kondapaka et al., 1997). Amplification of HER2 gene in breast cancer can range from 25 to 50 copies with up to 40 to 100 fold increase in HER 2 protein resulting in $2 \times 10^{\wedge} 6$ receptors expressed at the cell surface of the tumor (Iqbal and Iqbal, 2014). The dysregulation of HER 2 involves an excess of signals that stimulate cancer cells to grow and spread (Slamon et al., 1989).

\section{HER1 And HER2 detection}

Breast cancer can be classified based on its receptor status. This includes hormone receptor status such as progesterone (PR), estrogen (ER) receptor and epithelial growth factor receptor 2 (EGFR2/HER2). These receptors can be used as therapeutic targets as well as prognostic markers (Schulz et al., 2009; Brennan and Lim, 2015). Three main approaches can be used to identify the type

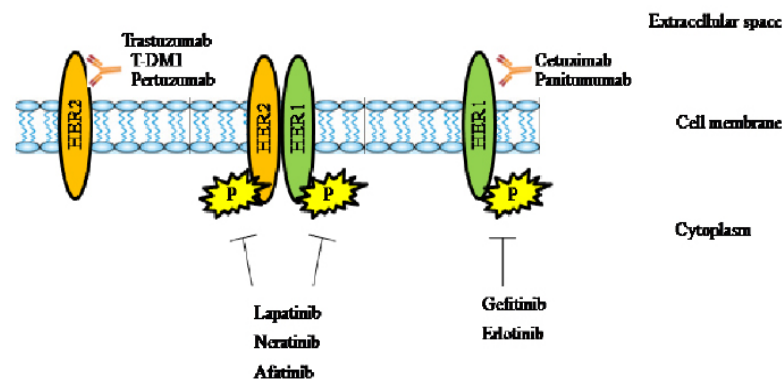

Figure 3. MAbs and TKIs Target HERs for the Treatment of Breast, Lung and Several other Types of Cancer

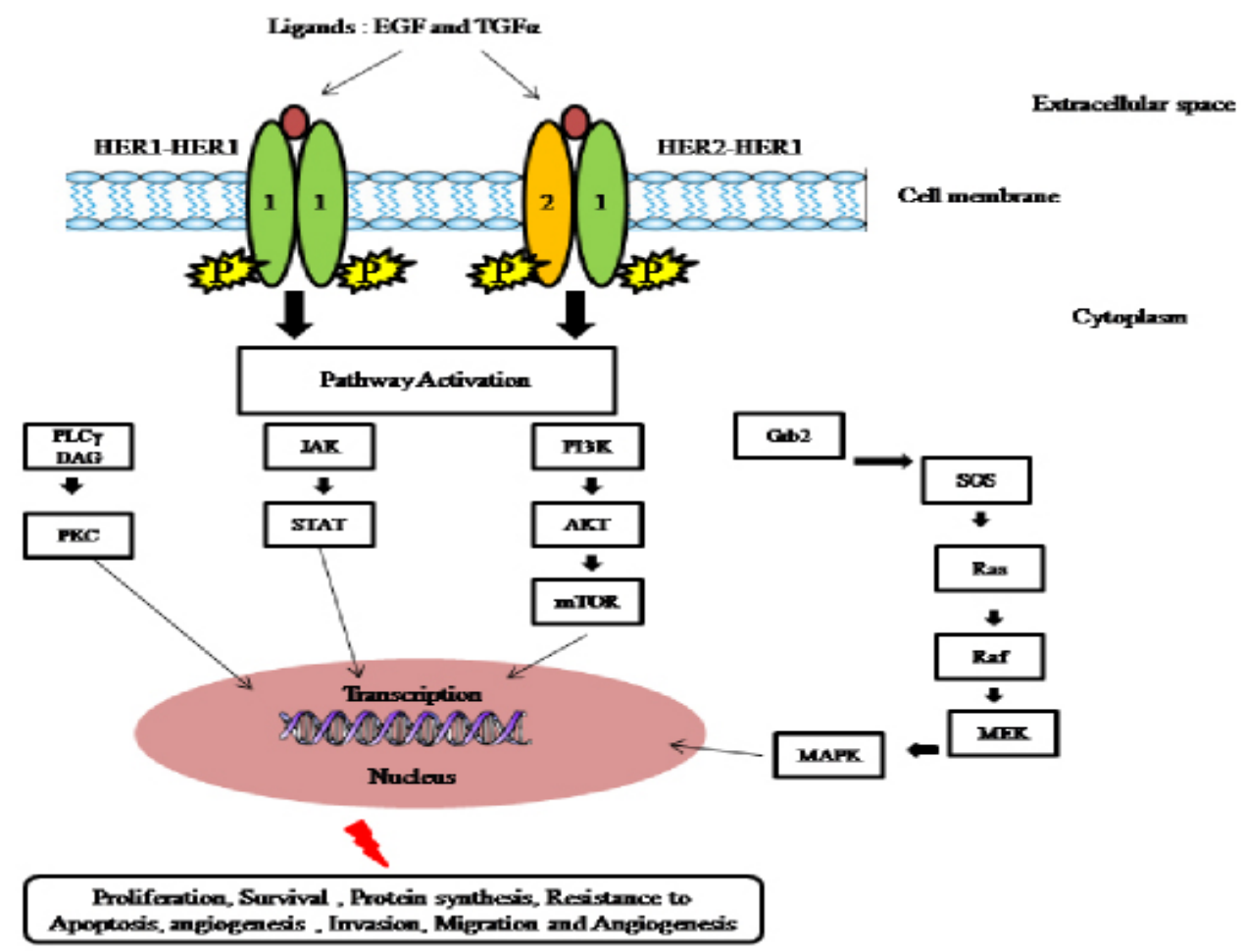

Figure 2. Homodimers and Heterodimers with the HER1 and, and Subsequent Stimulation of Downstream Signaling Pathways Such as PI3K/Akt, Ras/ Raf/Mek/Erk, PLC $\gamma$ 1/PKC, and JAK-STAT 
of breast cancer based on the receptor status. One of these approaches utilize immunohistochemistry (IHC) assay that measures the receptor status in tumors (Zaha, 2014). IHC is capable of measuring the production of HER2 protein in tumor (Menard et al., 2003; Schulz et al., 2009). Several antibodies have been used to determine the over expression of HER2 such as CB11 clone (Rhodes et al., 2002; Madrid and Lo, 2004; Iorio et al., 2005), rabbit anti-human polyclonal antibody A0485 (Gancberg et al., 2002; Rhodes et al., 2002; Schulz et al., 2009) and HercepTest, while the expression of HER 1 can be detected using monoclonal antibody $31 \mathrm{~g} 7$. All of these antibodies have been approved by United States Food and drug Administration (U.S. FDA) for therapeutic applications(Marquez et al., 2004). The scoring system of IHC is based on the staining intensity of tumor cells ranged between 0 and 3+ (Gancberg et al., 2002; Rhodes et al., 2002).

The staining score of HER 2 tumor cells is $2+$ or $3+$ while non HER2 tumor cells score is between 0 and 1+ (Madrid and Lo, 2004). IHC approach is faster and more economical to use but it could account for the some false-positive results that have to be verified with another approach such as fluorescence in situ hybridization (FISH) (Rhodes et al., 2002). FISH, which is a molecular method, is capable of detecting the level of HER2 gene amplification using fluorescent probes to look at the number of the HER2 gene copies (Madrid and Lo, 2004). Because the FISH technique is labor-intensive and expensive, Chromogenic in situ hybridization (CISH) can be used as a valid alternative to FISH. CISH is more economical to use and its method less cumbersome. CISH can be utilized to detect the over expression of HER 2 and HER 1 genes using a robust unique-sequence probe (Madrid and Lo, 2004; Marquez et al., 2004).

\section{HER1 and HER2 as therapeutic targets}

A range of approaches can be utilized to target HER1/ HER2 receptors including monoclonal antibodies (MAbs). These antibodies bind to the extracellular domain of the receptor and interfere with the binding of endogenous ligands that activate these receptors. Another class of compounds called small molecule inhibitors acts by binding at the intercellular tyrosine kinase domain to inhibit the kinase activity as shown in figure 3 . These approaches are mainly popular and explored for chemotherapy against cancers that overexpress HER 1 and HER 2 receptors. HER1 can be activated to initiate multiple

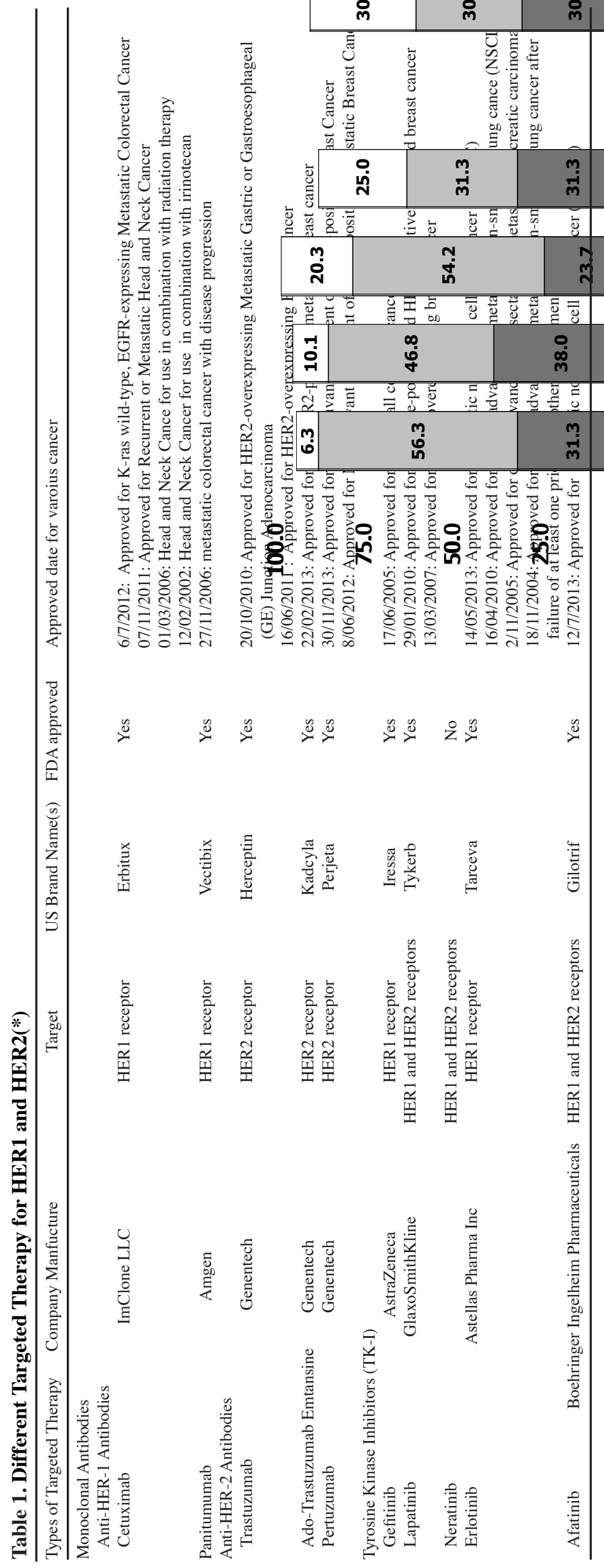

Asian Pacific Journal of Cancer Prevention, Vol 17, 2016 
downstream signaling pathways (Prenzel et al., 2001; Ettinger, 2006). Thus, HER1 signaling can be targeted in several ways either at the receptor level or by blocking the specific downstream pathway. To date there are no known ligands that can bind HER2 receptor (Harris et al., 2003). In addition HER2 receptor is the preferred dimerization partner for all HER family receptors (Tai et al., 2010). Consequently, several therapeutic drugs against HER2 targeting the extracellular domain and the intracellular tyrosine kinase domain as well as HER2 dimerization and downstream signaling pathways are in use or being developed (Prenzel et al., 2001; Schramm et al., 2015). Table 1 shows different targeted therapy including MAbs and small molecule inhibitorsfor HER1 and HER2.

\section{A-Monoclonal antibodies}

Anti-HER1 Antibodies: The use of HER1-specific MAbs to block HER1 receptor was the earliest approach for targeting aberrant HER1 signaling (Mendelsohn, 2000; Dhomen et al., 2012). Several ligands such as epidermal growth factor (EGF) and Transforming Growth Factor $\alpha$ (TGF $\alpha$ ) bind to HER1. Molecules such as MAbs bind to extracellular domain of HER1 and prevent these ligands from binding to HER1 with similar affinity that results in blocking the signaling cascade (Herbst and Bunn, 2003). MAbs against HER1 has been demonstrated to enhance antitumor activity in synergy with conventional anticancer therapies (Fan et al., 1993; Mendelsohn, 1997). Several MAbs has been developed for trageting HER1 such as Cetuximab and Panitumumab.

Cetuximabis an immunoglobulin (Ig) G1 humanmurine chimeric counterpart of the murine monoclonal antibody M225 which binds HER1with a 2-log higher affinity compared with the natural ligands EGF and TGF- $\alpha$ (Martinelli et al., 2009). Cetuximab binds to HER1 and promotes receptor internalization. Consequently, the receptor degradation occurs without receptor phosphorylation and activation. The down regulation of the receptor leads to the reduction of HER1 availability on the cell surface preventing the activation of HER1 and downstream signaling pathways (Martinelli et al., 2009). Cetuximab is capable of inhibiting the progression of cell cycle at the G0/G1 boundary and increasing the expression of cell cycle regulator p27KIP1 (Martinelli et al., 2009). Also, its antitumor activity can be attributed to its ability to induce apoptosis by increasing the expression of pro-apoptotic proteins such as caspase- 3 , caspase- 8 and caspase-9 (Mayo and Donner, 2001) or by inactivation of anti-apoptotic proteins such as Bcl-2 (Gingras et al., 1998). Cetuximab has been approved by FDA for the treatment of metastatic colorectal cancers with high level of HER1 expression. However, this drug has major side effects including high cost, short $\mathrm{t} 1 / 2$, toxicity, emergence of resistance (Foy et al., 2013), fever, nausea, elevation of liver enzymes skin reactions and allergies (Kamath and Buolamwini, 2006).

Panitumumab (ABX-EGF) is a fully human IgG2 monoclonal antibody targeting HER1 extracellular domains. It was developed by Abgenix's XenoMouse technology and offers effective high affinity therapy $\left(\mathrm{Kd}=5 \times 10^{-11} \mathrm{M}\right)$ with a minimum rate of anaphylaxis or allergic reactions (Mendez et al., 1997). It has higher binding affinity to HER1 receptor than cetuximab. Panitumumab unlike cetuximab, mediates its effect through mechanisms other than antibody-dependent cellular cytotoxicity (ADCC). Furthermore, it is being evaluated as monotherapy in patients with advanced solid tumors and metastatic colorectal cancer (CRC). Also, the combination of panitumumab with chemotherapy is used for various unresectable or recurrent cancers such as breast and ovarian cancers (Ettinger, 2006).

Anti-HER-2 Antibodies: HER 2 has been successfully targeted in different cancers including breast cancer (O'Sullivan et al., 2015). The extracellular domain of HER2 is being targeted in order to prevent receptor activation. A number of monoclonal antibodies such as trastuzumab and Pertuzumabwould bind to the extracellular domain of HER2 to suppress its activity by preventing phosphorylation of the tyrosine kinase domain and then preclude the initiation of downstream signaling pathways (Iqbal and Iqbal, 2014).

Trastuzumab (Herceptin) is a humanized monoclonal antibody that binds to the extracellular domain of HER2. A number of mechanism of Herceptin actions include inhibition of PI3K-AKT pathway, inhibition of HER2 shedding, attenuation of cell signaling, inhibition of tumor angiogenesis and antibody-dependent cellular cytotoxicity (Kute et al., 2004). However, Herceptin is not capable of preventing heterodimerization of HER2 with other HERs (Cho et al., 2003). Furthermore, Herceptin administration can result in several adverse effects with the most common being vomiting, diarrhea, fatigue, headache, rash, infusion reactions, neutropenia, and anemia (Iqbal and Iqbal, 2014). Additionally, serious undesirable effects include infusion reactions, febrile neutropenia, cardiomyopathy and pulmonary toxicity. Clinical trials in advanced metastatic breast cancer patients exhibit that the combination of Herceptin and Trastuzumabemtansine (T-DM1) which is an antibody drug conjugate composed of a maytansinoid derivative (a very potent microtubule destabilizing agent) is a vastly effective treatment with a very favorable safety profile (Burgess et al., 2014). T-DM1 inhibits cell signaling through the phosphatidylinositol 3-kinase/AKT pathway, inhibits HER2 shedding and induces antibodydependent cellular cytotoxicity (Welslau et al., 2014).

Ado-trastuzumabemtansine is an antibody-drug conjugate that comprise the monoclonal antibody trastuzumab connected to the cytotoxic agent mertansine (DM1). It offers a novel mechanism for overcoming trastuzumab resistance to HER2-positive metastatic breast cancer patients by utilizing trastuzumab to target the cytotoxic activity of DM1 to HER2 overexpressing cells. In a study by Verma et al., Ado-trastuzumabemtansine has been shown to extensively prolong progression-free as well as general survival with minimum toxicity than the combination of lapatinib and capecitabine(Verma et al., 2012). The United States FDA approved Adotrastuzumabemtansine for the treatment of HER2 positive metastatic breast cancer patients who earlier received trastuzumab and a taxane, either separately or in combination. However, this drug causes side effects such as elevated transaminases, constipation, headache, 
thrombocytopenia, fatigue, nausea, musculoskeletal pain and musculoskeletal pain (Amiri-Kordestani et al., 2014).

Pertuzumab is a humanized monoclonal antibody that binds the extracellular domain of HER2 and subsequently blocks the activation of the HER2 by hindering dimerization. The binding of Pertuzumab to HER2, unlike Herceptin, is predicted to block the region necessary for HER-2 dimerization with other HERs. Pertuzumab can prevent autocrine and pancrine produced ligands from inducing HER-2 on heterodimers (Agus et al., 2002). In addition, Pertuzumab is effective in treating tumors sensitive to Herceptin (Nahta et al., 2004). Clinical trials reveal that pertuzumab in combination with Herceptin and taxotere is greatly effective as a first-line treatment in patients with metastatic breast cancer (Burgess et al., 2014).

Tyrosine Kinase Inhibitors (TK-I): HER1 and HER2 tyrosine kinase domains are located within cell membrane which can be represented as a potential therapeutic target. Transphosphorylation and activation of the tyrosine kinase domain of HERs can be prevented using small molecules (Herbst and Bunn, 2003; Sergina et al., 2007). These molecules inactivate the tyrosine kinase domains which leads to suppression of downstream signaling pathways (Ettinger, 2006). Clinical research demonstrate that the level of phosphorlyation is associated with prognosis and hence making this target of interest (Kanematsu et al., 2003). Depending on the tyrosine residues of HER1and HER2 the site is either accessible or not, or has physicochemical properties that may be important enough for the design of specific inhibitors against diverse kinases. Several small molecule inhibitors including gefitinib (ZD1839, Iressa), erlotinib (OSI-774, Tarceva) and lapatinib have been developed and being used as antiHER1 and/or anti-HER2 therapeutic agents in cancers overexpressing these receptors.

Gefitinib has a small molecular weight and can inhibit HER1 tyrosine kinase activity with highly selective and reversible manner. It acts as competitive inhibitor of adenosine triphosphate binding to this receptor. Gefitinib is capable of inhibiting proliferation of cells overexpressing HER1 and HER2 by reduction of HER 1/HER2 heterodimer phosphorylation (Anderson et al., 2001). A number of studies reveal that $1 \mathrm{uM}$ of gefitinib treatment leads to the lossof phosphorylated HER1 in the acquired tamoxifen-resistant TAM-R cell line and inhibition of phosphorylation of the principle HER1 heterodimerization partner in these cells, HER2. This was demonstrated using western blotting and immunocytochemistry. The phosphorylation of MAPK was noticeably depleted as a result of this agent in TAM-R cells. This concentration of gefitinib reduced proliferation extensively by roughly $60 \%$ (Nicholson et al., 2002; Knowlden et al., 2003; Nicholson et al., 2004). This drug was approved by the U.S. FDA for clinical use in cancers overexpressing HER1.

Lapatinib, an orally active TKI which act as a potent dual inhibitor for HER2 and HER1 pathway. It was approved by U.S. FDA to be used in combination with capecitabine in patients who developed metastatic breast cancer and whose tumors overexpressed HER2, specifically who received previous therapy including trastuzumab, anthracycline and a taxane. This combination therapy was not associated with an increase in toxic side effects (Geyer et al., 2006). However, T-DM1 has superior efficiency and tolerability than capecitabine plus lapatinib (Welslau et al., 2014).

Neratinib is an oral irreversible TKI of HER2 and HER1. Like lapatinib, neratinib can enhance response of trastuzumab sensitive cell lines to trastuzumab and can overcome trastuzumab resistance in cell line models of obtained trastuzumab resistance. Also, neratinib has the ability to act on cell lines that are resistant to lapatinib and therefore, neratinib may have clinical benefit in patients who do not respond to lapatinib and/or trastuzumab (Canonici et al., 2013). Moreover, the combination of neratinib and capecitabine had a convenient toxicity profile and showed antitumor activity in HER2-positive metastatic breast cancer patients pretreated with trastuzumab and lapatinib (Saura et al., 2014).

Erlotinib is one of the TKI that was approved by USFDA for clinical use in cancers overexpressing HER1 and is able to inhibit HER1 with IC50 values in the 1-30 $\mathrm{nM}$ range (Kamath and Buolamwini, 2006). Recent study shows that the combination therapy of metformin and erlotinib may possibly have therapeutic efficacy in cancers driven by HER1 and PI3K signaling, including a subset of Basal-like breast cancers (BBC) patients and may provide a rationale for clinical study. Both of these are orally administered USFDA-approved drugs that can be translated into clinical trials and likely be an addition to other targeted drugs and chemotherapy (Lau et al., 2014).

Afatinib is also an oral, irreversible tyrosine kinase inhibitor targeting HER1 and HER2. It shows clinical activity in trastuzumab-refractory HER2-positive breast cancer. The phase one of the study shows that the maximum tolerated dose (MTD) of afatinib was 20 mg daily combined with the recommended weekly dose of trastuzumab, with $1 / 6$ patients (18 patients) showing dose-limiting toxicity (DLTs) in the dose escalation. However, this MTD cannot be recommended for phase II development without stringent diarrhea management. Therefore, further investigation is required for managing diarrhea (Ring et al., 2014).

MAbs and TKIs have been identified and approved for treatment of patients with breast cancer. However, the responders acquire resistance almost without exception. Small population of tumor cells called Breast cancer stem cells (BCSCs) play crucial role in initiation, propagation, resistance to therapy and recurrence of breast cancer. Therefore, these therapies could be more effective and cause few side effects if these cells are targeted.

\section{Breast Cancer Stem Cells (BCSCs)}

Stem cells have the ability of self-renewal. Cell division in a stem cell leads to the production of at least one daughter cell that has the same characteristics of the parent (Dragu et al., 2015). In general, stem cells are quiescent and may divide asymmetrically to generate another stem cell and one transit-amplifying cell. This provides continuation of the stem cell and the initial 


\section{Ibrahim O Alanazi and Zahid Khan}

material for production of differentiated cells (Morrison et al., 2008). Understanding the signaling pathways that are involved in the maintenance of cell division and differentiation of stem cells possibly will lead to greater insights about cancer stem cells capability of exponential long-term proliferation, self-renewal, and multipotency. The plasticity of stem cells permits them for the renewal of several lineages of differentiated cells during homeostasis. Normal and cancer stem cells share the property of expression of the ATP-binding cassette (ABC)-G2 transporter ABCG2 (Morrison et al., 2008) but ABCG2 does not express in most non stem cells (Dick, 2003). ABCG2 has the ability to pump out a variety of cell substrates such as cytotoxic drugs, using ATP. Cancer stem cells (CSCs) can be protected from cytotoxic drugs that are being used for cancer treatment by the high expression of ABCG2 transporters (Morrison et al., 2008). Thus, CSCs may contribute to therapeutic resistance (Morrison et al., 2008). Breast cancer stem cells (BCSCs) like other stem cells share the same property of slow-cycling and asymmetric cell division (Morrison et al., 2008).

\section{BrCa-IC / BCSCs detection}

The identification of breast cancer initiating cells (BrCa-IC) or (BCSCs) was obtained using a reliable NOD/SCID xenoexplant assay. According to Al-Hajj et al, the injection of single suspension of 100 enriched human breast cancer stem cells into the mammary fat pad of nonobese diabetic/severe combined immunodeficiency disease (NOD/SCID) mice, which are more immunedeficient than nude mice, causes these cells to grow tumors (Al-Hajj et al., 2003; Dick, 2003; Morrison et al., 2008). Four cell surface markers including adhesion molecules CD44 and CD24 (Dick, 2003; Dontu, 2008), a breast/ovarian cancer specific marker B38.1 and epithelial specific antigen (ESA) with heterogeneous expression in breast cancer tissue can be used for the identification of BrCa-IC (Dick, 2003). A study by Al-Hajj .et al, indicates that mice injected with CD44+, B38.1+ or CD24-/low generates tumors with $100 \%$ efficiency while none of the CD44- and B38.1-injections can cause tumors (AlHajj et al., 2003). In addition, lineage markers for all breast cancer tissue including hematopoietic, endothelial, mesothelial and fibroblast cells (Lin+) were used to show that only breast cancer tissue (Lin-) contains BrCa-IC. Lin- CD44+CD24-/low are divided depending on the expression of epithelial surface antigen (ESA). It has been found that Lin-ESA+CD44+CD24-/low cells can form tumors in NOD/SCID mice. Besides these four markers, there are several other markers that can be used to identify BCSCs (Dick, 2003). Several signaling pathways play a role in the characteristics displayed by CSCs including HER1 signaling (Li et al., 2008). The study performed by Li et al, suggests that HER1 signaling is required for cancer initiating cells self-renewal and that HER2positive cancers have increased self-renewal properties, suggesting that these tumorigenic cells may be susceptible to therapeutic agents targeting this signaling pathway in vivo (Li et al., 2008).

\section{Targeting stem cells for elimination}

Cancer therapy focus on targeting specific markers on tumor cells in order to stop the development of the tumor. These markers are overexpressed or mutated and often stand for genes, proteins or pathways considered to be crucial for the development of the tumor. For instance, Herceptin targets the HER2 protein. A number of studies show that HER2 gene overexpression accounts for around 20-30\% (Baselga, 2001; Basu, 2008; Korkaya et al., 2008; Morrison et al., 2008) of human breast cancers and is associated with aggressive metastatic disease. HER2 has the ability to regulate several signaling pathways such as $\mathrm{PI} 3 \mathrm{~K} / \mathrm{Akt}$. The contribution of this signaling in causing aggressive tumors is still unknown (Korkaya et al., 2008).

The stem/progenitor cell population can be identified using Aldefluor assay or immunohistochemistry that are capable of determining increased expression of the enzyme aldehyde dehydrogenase (ALDH1) in both the normal mammary gland and mammary carcinomas. Ginestier et al observed that there is a significant correlation between over expression of HER2 and expression of ALDH1, a stem cell marker, in a large number of breast cancer patients (Ginestier et al., 2007). The regulation of mammary stem cell population may be affected by the overexpression of HER2. This overexpression leads to a rise in the population of stem/progenitor cells of both normal tissue and malignant mammary tumors. CSCs or tumor stem cells are able to mediate tumorgenesis, invasion and metastasis as an effect of HER2 overexpression (Korkaya et al., 2008).

There is a correlation between the overexpression of HER2 and Akt phosphorylation. HER2 over expression leads to the enhancing of the phosphorylation of Akt in Aldefluor-positive HER2 stem cells. Herceptin has the ability to inhibit the overexpression of HER2 and AKT phosphorylation in sensitive but not in resistant cells. Hasan et al noted that there is no measurable effect on phospho-Akt and on phospho-HER 2 in herceptin resistant cells (Korkaya et al., 2008). They propose that the PI3K/ Akt pathway plays a crucial role in mediating the effects of HER2 signaling in breast cancer. PI3K pathway has been identified as the main determinant of herceptin resistant in breast cancer. Akt signaling also has a role in stem cell selfrenewal. New targets for cancer prevention and therapy can be obtained if the components of these pathways are identified (Korkaya et al., 2008). A number of approaches are being developed to target CSCs, however, no single approach has proven efficient (Dragu et al., 2015). The most crucial concerns that limit CSCs targeted therapeutics are intra-tumoral heterogeneity and potential toxicity to normal stem cells (Park et al., 2009). A recent study shows that the metallofullerenol nanomaterial Gd@C82(OH)22, has an intrinsic inhibitory activity against triple-negative breast cancer cells (TNBC) and is non toxic to normal mammary epithelial cells. Moreover, Gd@C82(OH)22 has the ability to block epithelial-to-mesenchymal transition with resultant efficient elimination of CSCs resulting in abrogation of tumor initiation and metastasis. Therefore, $\mathrm{Gd} @ \mathrm{C} 82(\mathrm{OH}) 22$ is recognized as non-toxic CSC specific inhibitors with considerable therapeutic potential (Liu et al., 2015). 


\section{Conclusions}

Targeted cancer therapy has made significant progress due to better understanding of carcinogenesis as a result of technological advancement in the field of cancer biology. A number of therapeutic agents directed against HER1 and HER2 have offered limited but promising alternatives to traditional chemotherapy improving the outcome for cancers overexpressing these tyrosine kinase receptors. These agents includes MAbs and tyrosine kinase inhibitors which have anti-tumor activity with satisfactory toxicity profiles. However, in majority of the cases there is relapse of the tumor probably due to the presence of therapy resistant CSCs. The recently identified metallofullerenol nanomaterial that is capable of eliminating CSCs while non toxic to normal tissue holds great promise in treating tumors that develop resistance attributable to its stem cell component. The use of anti-HER signal transduction drugs in combination with other anticancer agents requires additional investigations to determine clinical outcomes of anti-HERs therapy.

\section{Acknowledgements}

We acknowledge Professor Daved Adelson at Adelaide University for his critical comments in the writing of this review.

\section{References}

Agus DB, Akita RW, Fox WD, et al (2002). Targeting ligandactivated ErbB2 signaling inhibits breast and prostate tumor growth. Cancer Cell, 2, 127-37.

Al-Hajj M, Wicha MS, Benito-Hernandez A, et al (2003). Prospective identification of tumorigenic breast cancer cells. Proc Natl Acad Sci USA, 100, 3983-8.

Amiri-Kordestani L, Blumenthal GM, Xu QC, et al (2014). FDA approval: ado-trastuzumab emtansine for the treatment of patients with HER2-positive metastatic breast cancer. Clin Cancer Res, 20, 4436-41.

Anderson NG, Ahmad T, Chan K, et al (2001). ZD1839 (Iressa), a novel epidermal growth factor receptor (EGFR) tyrosine kinase inhibitor, potently inhibits the growth of EGFR-positive cancer cell lines with or without erbB2 overexpression. Int J Cancer, 94, 774-82.

Baselga J (2001). Phase I and II clinical trials of trastuzumab. Ann Oncol, 12, 49-55.

Basu A (2008). Molecular targets of breast cancer: AKTing in concert. Breast Cancer: Basic Clin Res, 2, 11-6.

Bouchalova K, Cizkova M, Cwiertka K, et al (2009). Triple negative breast cancer--current status and prospective targeted treatment based on HER1 (EGFR), TOP2A and C-MYC gene assessment. Biomedical papers of the Medical Faculty of the University Palacky. Olomouc, Czechoslovakia, 153, 13-7.

Brand TM, Iida M, Li CR, et al (2011). The nuclear epidermal growth factor receptor signaling network and its role in cancer. Discovery Medicine, 66, 419-32.

Brennan M,Lim B (2015). The actual role of receptors as cancer markers, biochemical and clinical aspects: receptors in breast cancer. Adv Exp Med Biol, 867, 327-37.

Burgess AW, Henis YI, Hynes NE, et al (2014). EGF receptor family: twisting targets for improved cancer therapies.
Growth Factors, 32, 74-81.

Canonici A, Gijsen M, Mullooly M, et al (2013). Neratinib overcomes trastuzumab resistance in HER2 amplified breast cancer. Oncotarget, 4, 1592-605.

Carpenter G, Cohen S (1979). Epidermal growth factor. Annu Rev Biochem, 48, 193-216.

Cho HS, Mason K, Ramyar KX, et al (2003). Structure of the extracellular region of HER2 alone and in complex with the Herceptin Fab. Nature, 421, 756-60.

Dhomen NS, Mariadason J, Tebbutt N, et al (2012). Therapeutic targeting of the epidermal growth factor receptor in human cancer. Crit Rev Oncog, 17, 31-50.

Dick JE (2003). Breast cancer stem cells revealed. Proc Natl Acad Sci USA, 100, 3547-9.

Dimitrakopoulos FI, Kottorou A, Antonacopoulou AG, et al (2015). Early-stage breast cancer in the elderly: confronting an old clinical problem. J Breast Cancer, 18, 207-17.

Dontu G (2008). Breast cancer stem cell markers - the rocky road to clinical applications. Breast cancer research : BCR, 10, 110 .

Dragu DL, Necula LG, Bleotu C, et al (2015). Therapies targeting cancer stem cells: Current trends and future challenges. World J Stem Cells, 7, 1185-201.

Ennis BW, Lippman ME, Dickson RB (1991). The EGF receptor system as a target for antitumor therapy. Cancer Invest, $\mathbf{9}$, 553-62.

Ettinger DS (2006). Clinical implications of EGFR expression in the development and progression of solid tumors: focus on non-small cell lung cancer. Oncologist, 11, 358-73.

Fan Z, Baselga J, Masui H, et al (1993). Antitumor effect of antiepidermal growth factor receptor monoclonal antibodies plus cis-diamminedichloroplatinum on well established A431 cell xenografts. Cancer Res, 53, 4637-42.

Foy KC, Wygle RM, Miller MJ, et al (2013). Peptide vaccines and peptidomimetics of EGFR (HER-1) ligand binding domain inhibit cancer cell growth in vitro and in vivo. $J$ Immunol, 191, 217-27.

Gancberg D, Jarvinen T, di Leo A, et al (2002). Evaluation of HER-2/NEU protein expression in breast cancer by immunohistochemistry: an interlaboratory study assessing the reproducibility of HER-2/NEU testing. Breast Cancer Res Treat, 74, 113-20.

Geyer CE, Forster J, Lindquist D, et al (2006). Lapatinib plus capecitabine for HER2-positive advanced breast cancer. $N$ Engl J Med, 355, 2733-43.

Ginestier C, Hur MH, Charafe-Jauffret E, et al (2007). ALDH1 is a marker of normal and malignant human mammary stem cells and a predictor of poor clinical outcome. Cell Stem Cell, 1, 555-67.

Gingras AC, Kennedy SG, O'Leary MA, et al (1998). 4E-BP1, a repressor of mRNA translation, is phosphorylated and inactivated by the $\mathrm{Akt}(\mathrm{PKB})$ signaling pathway. Genes Dev, 12, 502-13.

Harris RC, Chung E, Coffey RJ (2003). EGF receptor ligands. Exp Cell Res, 284, 2-13.

Herbst RS, Bunn PA, Jr. (2003). Targeting the epidermal growth factor receptor in non-small cell lung cancer. Clin Cancer Res, 9, 5813-24.

Huang L, Fu L (2015). Mechanisms of resistance to EGFR tyrosine kinase inhibitors. Acta Pharm Sin B, 5, 390-401.

Iorio MV, Ferracin M, Liu CG, et al (2005). MicroRNA gene expression deregulation in human breast cancer. Cancer Res, 65, 7065-70.

Iqbal N, Iqbal N (2014). Human epidermal growth factor receptor 2 (HER2) in cancers: overexpression and therapeutic implications. Mol Biol Int, 2014, 852748.

Kamath S, Buolamwini JK (2006). Targeting EGFR and HER- 
2 receptor tyrosine kinases for cancer drug discovery and development. Medicinal Res Reviews, 26, 569-94.

Kanematsu T, Yano S, Uehara H, et al (2003). Phosphorylation, but not overexpression, of epidermal growth factor receptor is associated with poor prognosis of non-small cell lung cancer patients. Oncol Res, 13, 289-98.

Knowlden JM, Hutcheson IR, Jones HE, et al (2003). Elevated levels of epidermal growth factor receptor/c-erbB2 heterodimers mediate an autocrine growth regulatory pathway in tamoxifen-resistant MCF-7 cells. Endocrinology, 144, 1032-44.

Kondapaka SB, Fridman R, Reddy KB (1997). Epidermal growth factor and amphiregulin up-regulate matrix metalloproteinase-9 (MMP-9) in human breast cancer cells. Int J Cancer, 70, 722-6.

Korkaya H, Paulson A, Iovino F, et al (2008). HER2 regulates the mammary stem/progenitor cell population driving tumorigenesis and invasion. Oncogene, 27, 6120-30.

Kute T, Lack CM, Willingham M, et al (2004). Development of Herceptin resistance in breast cancer cells. Cytometry A, 57, 86-93.

Lau YK, Du X, Rayannavar V, et al (2014). Metformin and erlotinib synergize to inhibit basal breast cancer. Oncotarget, 5, 10503-17.

Li X, Lewis MT, Huang J, et al (2008). Intrinsic resistance of tumorigenic breast cancer cells to chemotherapy. J Natl Cancer Inst, 100, 672-9.

Liu Y, Chen C, Qian P, et al (2015). Gd-metallofullerenol nanomaterial as non-toxic breast cancer stem cell-specific inhibitor. Nat Commun, 6, 5988

Lyon, France (2013). International Agency for Research on Cancer.

Madrid MA, Lo RW (2004). Chromogenic in situ hybridization (CISH): a novel alternative in screening archival breast cancer tissue samples for HER-2/neu status. Breast Cancer Res, 6, 593-600.

Marquez A, Wu R, Zhao J, et al (2004). Evaluation of epidermal growth factor receptor (EGFR) by chromogenic in situ hybridization (CISH) and immunohistochemistry (IHC) in archival gliomas using bright-field microscopy. Diagnostic Molec Path, 13, 1-8.

Martinelli E, De Palma R, Orditura M, et al (2009). Antiepidermal growth factor receptor monoclonal antibodies in cancer therapy. Clin Exp Immunol, 158, 1-9.

Mayo LD, Donner DB (2001). A phosphatidylinositol 3-kinase/ Akt pathway promotes translocation of $\mathrm{Mdm} 2$ from the cytoplasm to the nucleus. Proc Natl Acad Sci USA, 98, 11598-603.

Menard S, Pupa SM, Campiglio M, et al (2003). Biologic and therapeutic role of HER2 in cancer. Oncogene, 22, 6570-8.

Mendelsohn J (1997). Epidermal growth factor receptor inhibition by a monoclonal antibody as anticancer therapy. Clin Cancer Res, 3, 2703-7.

Mendelsohn J (2000). Blockade of receptors for growth factors: an anticancer therapy--the fourth annual joseph $\mathrm{H}$ burchenal american association of cancer research clinical research award lecture. Clin Cancer Res, 6, 747-53.

Mendez MJ, Green LL, Corvalan JR, et al (1997). Functional transplant of megabase human immunoglobulin loci recapitulates human antibody response in mice. Nature Genetics, 15, 146-56.

Miki Y, Swensen J, Shattuck-Eidens D, et al (1994). A strong candidate for the breast and ovarian cancer susceptibility gene BRCA1. Science, 266, 66-71.

Morrison BJ, Schmidt CW, Lakhani SR, et al (2008). Breast cancer stem cells: implications for therapy of breast cancer. Breast Cancer Res, 10, 210.
Nahta R, Hung MC, Esteva FJ (2004). The HER-2-targeting antibodies trastuzumab and pertuzumab synergistically inhibit the survival of breast cancer cells. Cancer Res, 64, 2343-6.

Nicholson RI, Hutcheson IR, Harper ME, et al (2002). Modulation of epidermal growth factor receptor in endocrine-resistant, estrogen-receptor-positive breast cancer. Ann N Y Acad Sci, 963, 104-15.

Nicholson RI, Hutcheson IR, Knowlden JM, et al (2004). Nonendocrine pathways and endocrine resistance: observations with antiestrogens and signal transduction inhibitors in combination. Clin Cancer Res, 10, 346-54.

Normanno N, De Luca A, Bianco C, et al (2006). Epidermal growth factor receptor (EGFR) signaling in cancer. Gene, 366, 2-16.

O'Sullivan CC, Bradbury I, Campbell C, et al (2015). Efficacy of Adjuvant Trastuzumab for Patients With Human Epidermal Growth Factor Receptor 2-Positive Early Breast Cancer and Tumors $</=2 \mathrm{~cm}$ : A Meta-Analysis of the Randomized Trastuzumab Trials. J Clin Oncol, 33, 2600-8.

Park CY, Tseng D, Weissman IL (2009). Cancer stem celldirected therapies: recent data from the laboratory and clinic. Mol Ther, 17, 219-30.

Prenzel N, Fischer OM, Streit S, et al (2001). The epidermal growth factor receptor family as a central element for cellular signal transduction and diversification. Endocr Relat Cancer, 8, 11-31.

Rhodes A, Jasani B, Anderson E, et al (2002). Evaluation of HER-2/neu immunohistochemical assay sensitivity and scoring on formalin-fixed and paraffin-processed cell lines and breast tumors: a comparative study involving results from laboratories in 21 countries. Am J Clin Pathol, 118, 408-17.

Ring A, Wheatley D, Hatcher H, et al (2014). Phase I study to assess the combination of afatinib with trastuzumab in patients with advanced or metastatic HER2-positive breast cancer. Clin Cancer Res, 21, 2737-44

Ruddon RW 2007. Cancer Biology, Oxford, Oxford University Press Inc.

Saura C, Garcia-Saenz JA, Xu B, et al (2014). Safety and efficacy of neratinib in combination with capecitabine in patients with metastatic human epidermal growth factor receptor 2-positive breast cancer. J Clin Oncol, 32, 3626-33.

Schramm A, De Gregorio N, Widschwendter P, et al (2015). Targeted therapies in HER2-positive breast cancer - a systematic review. Breast Care, 10, 173-8.

Schulz DM, Bollner C, Thomas G, et al (2009). Identification of differentially expressed proteins in triple-negative breast carcinomas using DIGE and mass spectrometry. J Proteome Res, 8, 3430-8.

Sergina NV, Rausch M, Wang D, et al (2007). Escape from HER-family tyrosine kinase inhibitor therapy by the kinaseinactive HER3. Nature, 445, 437-41.

Shiovitz S, Korde LA (2015). Genetics of breast cancer: a topic in evolution. Ann Oncol, 26, 1291-9.

Slamon DJ, Clark GM, Wong SG, et al (1987). Human breast cancer: correlation of relapse and survival with amplification of the HER-2/neu oncogene. Science, 235, 177-82.

Slamon DJ, Godolphin W, Jones LA, et al (1989). Studies of the HER-2/neu proto-oncogene in human breast and ovarian cancer. Science, 244, 707-12.

Tai W, Mahato R, Cheng K (2010). The role of HER2 in cancer therapy and targeted drug delivery. J Control Release, 146, 264-75.

Twelves CJ, Stebbing J (2012). Being there for women with metastatic breast cancer. a pan-European patient survey. Ann Oncol, 23, 132-. 
Valdehita A, Carmena MaJ, Bajo AM, et al (2012). RNA interference-directed silencing of VPAC1 receptor inhibits VIP effects on both EGFR and HER2 transactivation and VEGF secretion in human breast cancer cells. Molec Cellular Endocrinol, 348, 241-6.

Vandevijver MJ, Peterse JL, Mooi WJ, et al (1988). Neu-protein overexpression in breast-cancer - association with comedotype ductal carcinoma insitu and limited prognostic value in stage-II breast-cancer. N Engl J Med, 319, 1239-45.

Verma S, Miles D, Gianni L, et al (2012). Trastuzumab emtansine for HER2-positive advanced breast cancer. The New England journal of medicine, 367, 1783-91.

Welslau M, Diéras V, Sohn J-H, et al (2014). Patient-reported outcomes from EMILIA, a randomized phase 3 study of trastuzumab emtansine (T-DM1) versus capecitabine and lapatinib in human epidermal growth factor receptor 2-positive locally advanced or metastatic breast cancer. Cancer, 120, 642-51.

Zaha DC (2014). Significance of immunohistochemistry in breast cancer. World J Clin Oncol, 5, 382-92. 